\title{
Bioelectricity: Reasons to Be Amazed and Hopeful- My Personal Favorites
}

\author{
Dany Spencer Adams, PhD
}

\begin{abstract}
The field of bioelectricity is growing so rapidly, any attempt on my part to summarize its extraordinary breadth and depth would be a shallow report, a list really. Instead, I've zoomed in to introduce two marvelous examples of new understanding and application of bioelectricity. The signaling Venus flytraps use to capture and digest insects is a marvel of electrical engineering. The application of magnetic fields to treat conditions with brain involvement can offer relief from debilitating symptoms that have been refractory to other treatments. These, and a multitude of other fascinating stories, are helping to spread the word that bioelectricity can and should be seen as a critical approach that can change both science and medicine. This important journal will continue to carry that message.
\end{abstract}

Keywords: Venus flytrap, transcranial magnetic stimulation, treatment for depression, plant bioelectricity

$\mathbf{W}$ HEN I STARTED to get serious about being a professional biologist, few in my field of "developmental biology" wanted to think about, let alone study, physical phenomena. The researchers who did study the role of physical mechanisms in biology, both biomechanics and bioelectricity, knew that biophysics is actually quite interesting, not to mention important and powerful. The current rise in the number of scientists and physicians studying biological and medical questions from a bioelectric perspective has kept me amazed and made me hopeful.

The variety of questions and goals described in our young journal Bioelectricity during its first year testifies to the rapidly spreading interest in the roles of ions, membrane voltage, current and resistance, and their control during biological processes. More special issues have been planned: Methodology of Research and Applications of Electric Fields, notably microfluidic devices, computational models, and novel therapies; and Electrically Charged Biomaterials for Drug Delivery and Tissue Repair, including tissue scaffolding, biomaterials, and implant coatings (see www .liebertpub.com/bioe for further information). These will bring researchers and physicians up to date on the status of particular directions and questions, and inspire new studies and applications. From the creation of microbial fuel cells to developments in regenerative medicine and cancer, bioelectricity is increasingly being recognized as one of the fundamental mechanisms underlying all of biology, informing researchers, engineers, and health care professionals.
I cannot begin to address all of the topics under study by researchers in the field of bioelectricity, although I can recommend searching for "bioelectric*" in Pubmed to get a sense of the breadth and depth of work in this field, as well as the recent exponential increase in articles. Instead, I summarize here two examples of bioelectricity in action, favorites of mine, at least among those favorites that have not yet appeared in these pages.

\section{The Venus Flytrap}

For pure intellectual pleasure, there are the mechanisms underlying how Venus flytraps capture insects (Fig. 1). Videos of the process are like horror films; you want to yell "run away, don't go in there! You are going to be eaten alive!" And then the trap snaps closed. How the plant captures live insects, and not other things, is an amazing combination of interacting physical processes. ${ }^{1}$ Lured by an aroma (composed of $>60$ volatile organic compounds), and by the captivating red color that appears when the plant needs food, a meal lands on one of the specialized clam-shell-shaped leaf tips known as lobes. In addition to the spines along the edges that act like bars of a jail during the earliest stages of prey capture, there are three to four spines on the flat inner surface of each lobe called trigger hairs. At the base of each trigger hair, there are sensory cells that can create an action potential (AP). These APs involve $\mathrm{K}^{+}$channels, but not animal-like $\mathrm{Na}^{+}$channels that have not been found in plants. ${ }^{2}$ Also, unlike neurons, depolarization depends on anion efflux rather than cation influx.

Ion Diagnostics LLC, Watertown, Massachusetts. 


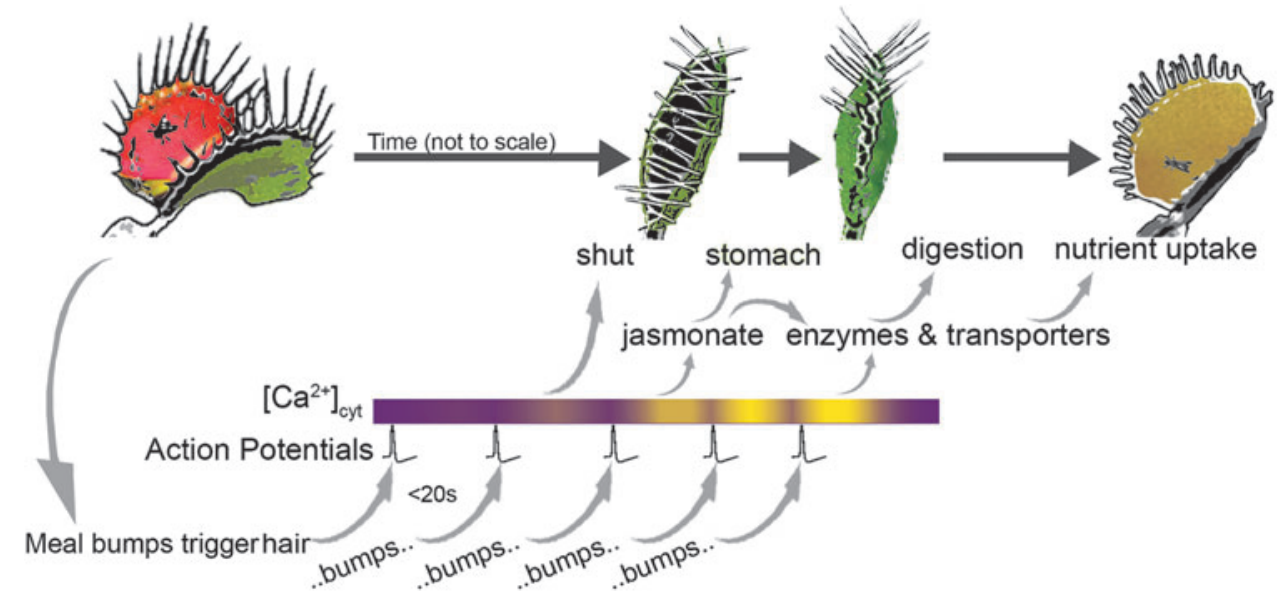

FIG. 1. Some of the bioelectric mechanisms by which Venus flytraps capture prey, then convert the lobes into a green stomach, and digest. Across the top is a representation of the changes to the lobes as they snap shut, seal to become a leakproof stomach, and produce the enzymes and transporters used for digestion and nutrient uptake. Across the bottom are five moments when the prey displaces a trigger hair, and thereby triggers an AP that leads to calcium release. When the APs are close enough together, the cytoplasmic calcium concentration $\left(\left[\mathrm{Ca}^{2+}\right]_{\text {cyt }}\right)$ increases over time (more yellow means more $\left.\left[\mathrm{Ca}^{2+}\right]_{c y t}\right)$. The gray arrows indicate positive regulation. AP, action potential.

These APs can trigger closing of the lobes only if there are two spikes within $20 \mathrm{~s}$ of each other; that is what prevents a nonmoving nonmeal from triggering the mechanism. But a meandering insect that bumps into a trigger hair within $20 \mathrm{~s}$ of the first bump sets off a series of events that, although not a domino-like cascade, nonetheless ensures that everything happens in the right order due to the "calcium clock." The ordering mechanism involves AP-induced transient increases of cytoplasmic $\mathrm{Ca}^{2+}$ concentration $\left(\left[\mathrm{Ca}^{2+}\right]_{\text {cyt }}\right)$; if the APs occur quickly enough, that is, before $\left[\mathrm{Ca}^{2+}\right]_{\text {cyt }}$ goes back to a minimum, each induced transient builds on the last, raising the $\left[\mathrm{Ca}^{2+}\right]_{\text {cyt }}$ to a new higher level. The downstream events each depends on a particular $\left[\mathrm{Ca}^{2+}\right]_{\text {cyt }}$; as the $\left[\mathrm{Ca}^{2+}\right]_{\text {cyt }}$ rises, the next event in the sequence is triggered. That second AP, within $20 \mathrm{~s}$ of the first, increases $\left[\mathrm{Ca}^{2+}\right]_{\text {cyt }}$ past the threshold that triggers closure of the lobes; it causes cells at the center of the lobe to rapidly lose water (another ion flux-dependent phenomenon) and that causes turgor pressure to plummet. Turgor pressure prevents the lobes from springing shut, the same way air pressure keeps a balloon inflated; when you let the air out, the elastic energy stored in the rubber shrinks the balloon back to its uninflated size. When water is suddenly released from the cells, elastic energy stored in the lobe (due to variations in ion concentrations in apposed cell layers) snaps the trap closed in $<0.1 \mathrm{~s}$.

Continued movement by the meal bumps the trigger hair(s) again and again causing $\left(\left[\mathrm{Ca}^{2+}\right]_{\mathrm{cyt}}\right)$ to reach new thresholds. Three APs leads to synthesis of jasmonate, a so-called touch hormone. Jasmonate stimulates the secretion of digestive enzymes and the complete sealing of the pocket to prevent escape and leakage, turning the pocket into a "green stomach" with digestive-enzyme secreting gland cells. More than five APs trigger expression of genes for the transporters needed to absorb the nutrients. That, to put it informally, is so cool.

I love this example because, although there is still much to learn, the physical mechanisms - to my mind, the bottomline explanations - are afforded the appropriate respect. This

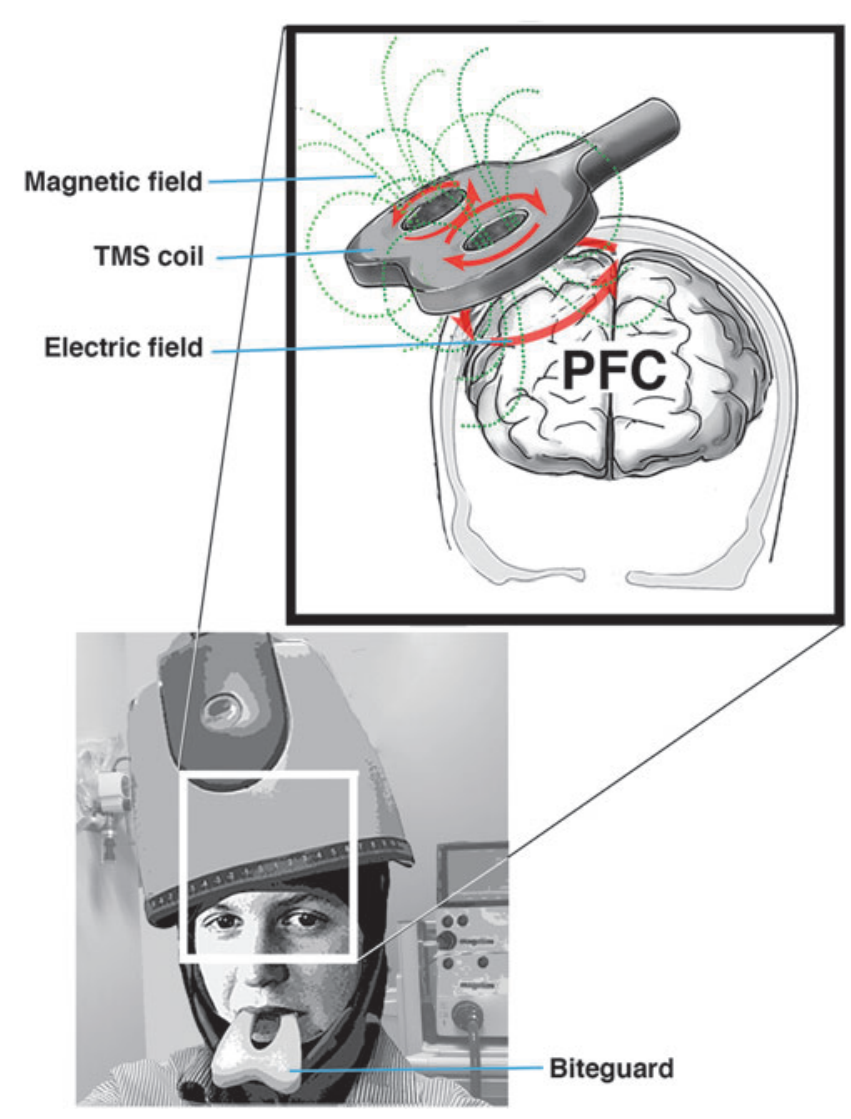

FIG. 2. Illustration of a patient receiving TMS delivered using a coil. For treatment of depression, the fields are aimed toward the left PFC. The bite guard is a single-use block that protects the teeth from the clenching that occurs during the 2-s activation of the magnetic field. The control system is monitored by a trained nurse who sits with the patient for the duration of the treatment. Inset after Vlachos et al. ${ }^{4}$ PFC, prefontal cortex; TMS, transcranial magnetic stimulation. 
fantastically coordinated series of events illustrate the interplay of mechanics and electricity with the more familiar chemical and molecular events, in an organism we have all watched with wonder and awe. It does not get much better than that.

\section{Transcranial Magnetic Stimulation}

A second favorite is "transcranial magnetic stimulation", (TMS). ${ }^{3}$ Less famous than its more dramatic cousin electroconvulsive therapy, over the past three decades TMS has been used to help patients with a variety of severe neurological conditions, including Parkinson's, strokes, epilepsy, and migraines. Compared with the Venus flytrap, we know very little about how TMS does what it does. We do know, however, that it has had success at relieving suffering. I know. I have had TMS.

Severe depression led me to TMS this past year. Remarkably, in two insurance-covered months, TMS brought an end to my own personal 30 years war, a blackness that varied in saturation, but still affected almost every day and every aspect of my life. I have found it impossible to explain what the difference feels like beyond "wow."

The business end of the TMS apparatus is a magnetic coil inside a helmet that is positioned on the patient's head (Fig. 2) so as to target a magnetic field/induced current to the relevant brain area. The response to the field depends on where it is aimed. If the target is the motor cortex, the field causes muscle contractions in the extremities. If it is the visual cortex, patients see flashes of light when their eyes are closed. One mode, known as repetitive TMS (rTMS), involves rapid alternation of the direction of the magnetic field, this is the mode currently used in clinical neurology. Interestingly, low frequency rTMS $(<1 \mathrm{~Hz})$ reduces neuronal excitability, whereas high frequency increases cortical excitability. Currently, it is thought that the longer term effects of rTMS also vary with the frequency and intensity of the stimulation.

The most widely accepted mechanistic hypothesis is that rTMS brings on a shift in neuronal excitability. This shift is caused by a change in the ionic environment surrounding the target neurons, leading to synaptic plasticity. The long-term effects of rTMS are thought to be due to that plasticity allowing change in long-term potentiation; evidence consistent with this hypothesis comes from studies in rodent hippocampus, although those results are highly dependent on the rate of stimulation.

It is believed that at the molecular level, long-term potentiation is brought about through depolarization-induced changes to the cation channel domain of the $N$-methyl D-aspartate receptor. The so-called late long-term potentiation (the kind that lasts and might, therefore, explain longterm therapeutic effects) is correlated with changes in gene expression and protein synthesis. This suggests a direct link between bioelectric events and the processes that would provide the building blocks needed for more permanent changes to the neurons. Another intriguing finding is that polymorphisms in one of the serotonin receptors $\left(5-\mathrm{HT}_{1 \mathrm{~A}}\right.$, a $\mathrm{G}$-protein, and $\mathrm{K}^{+}$channel) correlate with responsiveness to TMS, hinting at overlapping mechanisms of the selective serotonin reuptake inhibitor-type antidepressants and the antidepressive effects of TMS.

\section{Conclusion}

TMS, and other bioelectricity-dependent treatments, such as the tools described in the December 2019 Special Issue of Bioelectricity (Practical Applications of Bioelectric Stimulation), promises to revolutionize research, understanding, diagnostics, and therapeutics. Wherever there are chemical reactions, there is bioelectricity; the Venus flytrap is just one illustration of that fundamental truth. In fact, research is showing again and again that bioelectricity can regulate chemical reactions. Employing bioelectricity, rather than targeting a particular molecule that acts in a pathological process, apparently turns on not just the correct process, but its regulation as well. Starting a molecular cascade by influencing an early bioelectric step frequently leads to the process getting turned off at the correct time, too, a process that is critical but all too often forgotten. I can see a future in which bioelectricity is commonly utilized to trigger an organism's built-in healing processes, even those that, like regeneration in adults, are not normally active. While understanding those processes is fascinating and important research, it may be that medicine can also be served when we do not try to micromanage biochemistry, but, instead, convince the body to heal itself. I believe that bioelectricity is the language we need to learn. Bioelectricity can be our textbook.

\section{Author Disclosure Statement}

No competing financial interests exist.

\section{References}

1. Hedrich R, Neher E. Venus flytrap: How an excitable, carnivorous plant works. Trends Plant Sci 2018;23:220-234. DOI: $10.1016 /$ j.tplants.2017.12.004

2. Hedrich R. Ion channels in plants. Physiol Rev 2012;92: 1777-1811. DOI: 10.1152/physrev.00038.2011

3. Chervykov AV, Chernyavsky AY, Sinitsyn DO, et al. Possible mechanisms underlying the therapeutic effects of transcranial magnetic stimulation. Front Hum Neurosci 2015;9:303. DOI: 10.3389/fnhum.2015.00303

4. Vlachos A, Funke K, Ziemann U. Assessment and modulation of cortical inhibition using transcranial magnetic stimulation. Neuroforum 2017;23:A9-A17. DOI: 10.1515/nf-2016-A103

Address correspondence to: Dany Spencer Adams, PhD Ion Diagnostics LLC

200 Dexter Avenue, ste 260 Watertown, MA 02472

E-mail: Adams@Ion-Diagnostics.com 